\title{
Desarrollo de Ormosia schunkei en suelos recuperados con biocarbón activado en biol de vacaza
}

\author{
Development of Ormosia schunkei in recovered soils \\ with biochar activated in biol vaccine
}

\author{
Ronald Lozano ${ }^{1, \star}$, Wilder G. Nieto ${ }^{1}$, Mariano M. Mendoza ${ }^{1}$ y Fred C. Ramirez ${ }^{1}$
}

\begin{abstract}
Recibido: 20 julio 2020 | Aceptado: 23 setiembre 2020 | Publicado en línea: 12 diciembre 2020 Citación: Lozano, R; Nieto, WG; Mendoza, MM; Ramírez, FC. 2020. Desarrollo de Ormosia schunkei en suelos recuperados con biocarbón activado en biol de vacaza. Revista Forestal del Perú 35 (3, Número Especial): 18-27. DOI: http://dx.doi.org/10.21704/rfp.v35i3.1598
\end{abstract}

\begin{abstract}
Resumen
Ormosia schunkei (huairuro) es una especie forestal amazónica que las culturas indígenas utilizan en la producción de artesanía y es por esta razón que es muy valorada por comunidades locales, sin embargo, su población natural disminuye rápidamente debido a la deforestación, las prácticas insostenibles de producción maderera y la colecta comercial semillas. Por ello las plantaciones forestales son una opción para la producción sostenible de semilla en tierras deforestadas dentro de comunidades indígenas. Debido al nivel de degradación de las áreas disponibles para plantaciones forestales es necesario mejorar previamente las condiciones del suelo con tecnología accesibles localmente que permitan un adecuado crecimiento y desarrollo de la plantación. El objetivo de este experimento fue describir el efecto en el crecimiento de las plantaciones de O. shunkei con Biochart o Biocarbón producido con base en los desechos industriales de la producción de Palma aceitera, los cuales son muy abundantes en la zona de estudio. La evaluación indica que la aplicación de Biocarbón en los suelos promueve un mayor crecimiento inicial de la plantación y que la tasa de supervivencia fue $4 \%$ mayor en las parcelas fertilizadas a 90 días de la plantación. A pesar del mayor crecimiento y supervivencia inicial de la plantación objeto del tratamiento, recomendamos una evaluación a largo plazo para comprobar el efecto de esta enmienda en el crecimiento de la especie y las propiedades químicas del suelo.
\end{abstract}

Palabras clave: Huayruro, biocarbón, pirólisis, fermentación, biol, fertilizante

${ }^{1}$ Universidad Nacional Intercultural de la Amazonia-UNIA-Pucallpa-Perú

Autor de Correspondencia: rodmarlon2166@gmail.com 


\begin{abstract}
Ormosia schunkei (huairuro) is a tropical tree, amazon indigenous cultures use this specie for craft production and, therefore, is very valuable to local communities. However, natural population decrease due deforestation, unsustainable timber harvest and commercial seed collection. As a consequence, planting forests is a suitable option for sustainable production, and to recover deforested lands within indigenous communities. Because the soil degradation in areas available for forest plantations, is compulsory to improve soil condition before planting to allows effective growth of plantation. The objective of this experiment is describe the effect of Biochart or Biocarbon in forest plantation. This fertilizer is locally produced from abundant waste of oil palm production through intermediate technology. Biochart application to the soils reported a significant change in their chemical properties and fertility, and plantation shows superior growth in fertilized plots and survival rate was $4 \%$ higher on fertilized plots 90 days after planting. Despite initial superiority of growth and survival rate on plantation a long term evaluation is necessary to measure long term effects of this amendment on plantation and soil properties.
\end{abstract}

Key words: Huayruro, biochar, pyrolysis, fermentation, biol, fertilizer

\section{Introducción}

En la Amazonia es la vegetación arbórea la que contiene los nutrientes que se reciclan con gran velocidad y mantienen una vegetación exuberante. Los suelos amazónicos poseen baja fertilidad natural y sin vegetación son altamente ácidos, con un $\mathrm{pH}$ de 4,8 en suelos de bosques secundarios de 5 años y pH de 4,6 en suelos para bosques secundarios de 16 años (Ruíz y Davey 2005). Estas condiciones los hacen inadecuados para su aprovechamiento agropecuario convencional. Además, en zonas deforestadas de la Amazonia se pierden los nutrientes con gran facilidad debido a la alta precipitación, efecto de la intemperie y a la ausencia de vegetación que recicle los nutrientes.

La expansión de cultivos industriales, como Palma Aceitera, en áreas deforestadas es una de las opciones elegidas para recuperar capacidad productiva de estos suelos degradados. Actualmente en la Amazonia peruana se desarrollan empresas agroindustriales y pecuarias que generan residuos que pueden ser aprovechados mediante tecnologías apropiadas. Tal es el caso de los cuescos de palma aceitera y el estiércol de ganado vacuno (vacaza), los cuales al contener elementos lignocelulósicos, nutricionales y bióticos en su composición, son excelentes materias primas para elaborar compuestos mejoradores de la estructura del suelo y fertilizantes para promover el desarrollo de cultivos.
En razón de lo descrito, la pirolisis que es la degradación térmica de material orgánico a altas temperaturas en ausencia de oxígeno (Escola et al. 2014), puede ser empleado con material ligno celulósico de los cuescos de palma aceitera para mejorar las características agrícolas de suelos degradados; así como el estiércol vacuno, diluido y mediante una fermentación anaeróbica, se convierte en un excelente fertilizante líquido (biol) (Gutiérrez et al. 2018), pudiendo actuar como un promotor del crecimiento y desarrollo de especies forestales durante su cultivo, y permitiendo posteriormente que estas especies sean aprovechables para producir desde alimentos, medicinas, textiles, energía y artesanías.

Hoy en día, en la región ucayalina, y en muchas de sus poblaciones autóctonas, se desarrolla la actividad artesanal, como una tradición ancestral, usando como materia prima importante, las semillas de la O. schunkei (huairuro), a tal punto que se ha constituido en una actividad económica preponderante para lograr generar ingresos monetarios para las numerosas familias de artesanos. Sin embargo, la especie forestal aprovechada para estos fines está siendo explotada de modo no sostenible, llevando a la casi total pérdida de los ejemplares productores de semillas, por lo cual, su adquisición supone en elevados costos, pues debe ser transportada de lugares lejanos 
lo cual afecta el volumen de ventas y restringe la obtención de ganancias.

En ese sentido, se planteó como objetivo describir el efecto en el crecimiento y porcentaje de supervivencia de las plantaciones de $O$. shunkei con Biochart o Biocarbón producido aprovechando los cuescos de la industria de la Palma aceitera y enriquecido en biol de vacaza, los cuales son muy abundantes en la zona de estudio.

\section{Materiales y Métodos}

\section{Área de estudio}

La investigación fue desarrollada en la Comunidad Nativa de San Francisco, distrito de Yarinacocha, provincia de Coronel Portillo, región Ucayali a una altitud de 120 m.s.n.m; geográficamente se localiza entre las coordenadas UTM N9085272; E539574; Zona 18 L.

\section{Características edafo climáticas}

El área experimental presenta un clima cálido y lluvioso, con temperatura promedio anual de $26^{\circ} \mathrm{C}\left(21-38^{\circ} \mathrm{C}\right)$, precipitación pluvial anual de $2344 \mathrm{~mm}$, humedad anual media de 84,2\% (Guerrero et al. 2012).

El clima en la región Ucayali se caracteriza por ser tropical; corresponde a selva baja, con ecosistema de bosque tropical semisiempre verde estacional (Cochrane 1982).

\section{Elaboración y análisis de Biol de vacaza}

Se recolectó el estiércol de ganado vacuno el cual fue trasladado hacia el centro de acopio y elaboración. La elaboración se inició en el interior de un tanque de $1100 \mathrm{~L}$ previamente desinfectado por calor, al que se agregó estiércol de ganado vacuno (40\%), agua (40\%), cultivo láctico-Biolac (5\%) y melaza de caña de azúcar (15\%). Periódicamente se supervisó el proceso de degradación de la mezcla, por medio de la medición del $\mathrm{pH}$ hasta que alcanzó un pH de 3,77 en 63 días de fermentación anaeróbica, parámetro indicado por Peralta et al. (2016) como lo adecuado para este tipo de fertilizante. Posteriormente, el biol fue analizado en las instalaciones del Laboratorio de
Análisis de Suelos, Plantas, Aguas y Fertilizantes-LASPAF de la Universidad Nacional Agraria La Molina-UNALM.

\section{Fabricación, activación y caracterización del biocarbón}

El cuesco de palma aceitera se trasladó a la localidad de San Francisco, para luego ser sometido a pirólisis en carbonizadores tipo PhilRice estilo abierto. Después de 12 horas de carbonización en rangos de temperatura entre 159 y $490^{\circ} \mathrm{C}$, fue enfriado, envasado, molido a tamaños de $\leq 1,1,4$ y $2,0 \mathrm{~mm}$, mezclado con biol de vacaza y dejado en reposo dos días antes de su uso para su activación, siendo después caracterizados fisicoquímicamente como biocarbón y biocarbón enriquecido en el Laboratorio de Análisis de Suelos, Plantas, Aguas y Fertilizantes-LASPAF de la Universidad Nacional Agraria La Molina-UNALM.

\section{Preparación y manejo de la semilla de $\boldsymbol{O}$. schunkei}

Las semillas de O. schunkei, procedieron del rodal ubicado en la Comunidad Nativa de Fernando Sthal, distrito de Tahuanía, provincia de Atalaya, región Ucayali, las mismas que fueron primero escarificadas, para luego ser sumergidas en agua durante 24 horas, a fin de acelerar la germinación. Posteriormente, entre el día 17 al 33, los brinzales fueron repicados a bolsas de vivero, protegidas por malla al $80 \%$ de sombra y con aplicación de biol como fertilizante foliar. A 270 días de su introducción al vivero, fueron separadas en 2 grupos de 180 plantas cada uno y establecidas en 2 parcelas.

\section{Preparación del terreno y plantación de $O$. schunkei}

El terreno destinado a la plantación estaba invadido por Imperata brasiliensis Trin. (cashaucsha), la cual fue removida inicialmente mediante corte de motocultivadoras, para posteriormente aplicar labranza mecanizada y control químico de malezas. Este terreno se dividió en 2 parcelas (P1 y P2), siendo P1 la parcela testigo. A P2 se agregó el biocarbón en una dosis de 10 t.h ${ }^{-1}$, el cual se homogenizó con una remoción suave del suelo, dejando dos días de reposo para la adaptación del consorcio mi- 


\begin{tabular}{|c|c|}
\hline \multicolumn{2}{|c|}{ Dosis adecuada } \\
\hline Nutrientes & $\begin{array}{c}\text { Macronutrientes } \\
\text { g/kg }\end{array}$ \\
\hline $\mathrm{N}$ & $28-40$ \\
\hline $\mathrm{P}$ & $2,5-4$ \\
\hline $\mathrm{K}$ & $15-30$ \\
\hline $\mathrm{Ca}$ & $5-7$ \\
\hline $\mathrm{Mg}$ & $2-3$ \\
\hline $\mathrm{S}$ & $2-2,5$ \\
\hline Nutrientes & Micronutrientes \\
\hline $\mathrm{mg} / \mathbf{k g}$ \\
\hline $\mathrm{B}$ & $35-70$ \\
\hline $\mathrm{Fu}$ & $8-15$ \\
\hline $\mathrm{Mn}$ & $101-220$ \\
\hline $\mathrm{Zn}$ & $250-500$ \\
\hline & $30-60$ \\
\hline
\end{tabular}

Cuadro 1. Caracterización fisicoquímica del biol obtenido de estiércol vacuno.

\begin{tabular}{|l|c|}
\hline \multicolumn{1}{|c|}{ Característica } & Valor \\
\hline $\mathrm{pH}$ & 5,14 \\
\hline $\begin{array}{l}\text { Conductividad Eléctrica (CE), } \\
\mathrm{dS} / \mathrm{m}\end{array}$ & 2,33 \\
\hline Materia orgánica (MO), \% & 90,94 \\
\hline Nitrógeno (N), \% & 1,13 \\
\hline Fósforo (P), \% & 0,47 \\
\hline Potasio (K), \% & 0,36 \\
\hline Calcio (Ca), \% & 0,25 \\
\hline Magnesio (Mg), \% & 0,24 \\
\hline Sodio (Na), \% & 0,02 \\
\hline Fierro (Fe), ppm & 493,00 \\
\hline Cobre (Cu), ppm & 20,00 \\
\hline Zinc (Zn), ppm & 25,00 \\
\hline Manganeso (Mn), ppm & 151,00 \\
\hline Boro (B), ppm & 12,00 \\
\hline Cadmio (Cd), ppm & 0,48 \\
\hline Cromo (Cr), ppm & 12,48 \\
\hline
\end{tabular}

Cuadro 2. Características fisicoquímicas de los cuescos de palma aceitera. crobiano a las condiciones del suelo. Después de este reposo se inició la primera siembra de frijol caupí, con el fin de mejorar el contenido de nitrógeno orgánico, y luego de la segunda cosecha de vainas, a los 105 días, se procedió a la plantación de $O$. schunkei, empleando un distanciamiento de $5 \times 5 \mathrm{~m}$. Los plantones de la especie forestal fueron distribuidos en 20 filas y 9 columnas totalizando 180 plantas en cada una de las parcelas. Los plantones establecidos en los bordes se consideran no evaluables.

Las plantas de O. schunkei, se evaluaron a los 30, 60 y 90 días de plantados en cuanto a la ganancia de altura en $\mathrm{cm}$, y a los 90 días se determinó el porcentaje de supervivencia, a pesar de que se trata de una evaluación de crecimiento inicial.

\section{Diseño experimental}

Para determinar las diferencias entre tratamientos (T0 sin biocarbón y T1 con biocarbón) en la supervivencia se comparó el porcentaje de plantas vivas en cada parcela a los 90 días excluyendo los bordes, lo que da como resultados 18 filas y 7 columnas totalizando 126 plantas evaluables.

El diseño experimental para la comparación de altura fue el de bloques completamente al azar. La supervivencia y el crecimiento en altura de los plantones de O. shunkei fueron evaluados de diferente forma. Para evaluar las diferencias de crecimiento entre tratamientos, se seleccionaron al azar 10 plantas en la parcela testigo y 10 plantas al azar en la parcela con tratamiento. Mientras que para la evaluación de supervivencia se consideró 126 plantas, sin considerar las plantas de los bordes.

\section{Resultados}

\section{Caracterización del biol y cuescos de palma aceitera}

El biol evidenció características muy notorias en los tres elementos esenciales para el desarrollo de las plantas como nitrógeno-N (994 mg/L), fósforo-P $(336,31 \mathrm{mg} / \mathrm{L})$ y potasio-K (1 $325 \mathrm{mg} / \mathrm{L})$ así como materia orgánica-MO $(33,16 \mathrm{~g} / \mathrm{L})$ en solución, complemen- 


\begin{tabular}{|l|c|c|}
\hline \multirow{2}{*}{ Característica } & \multicolumn{2}{|c|}{ Valor } \\
\cline { 2 - 3 } & Biocarbón & $\begin{array}{c}\text { Biocarbón } \\
\text { enriquecido }\end{array}$ \\
\hline $\mathrm{pH}$ & 7,72 & 7,67 \\
\hline Conductividad Eléctrica (CE), dS/m & 0,99 & 3,14 \\
\hline Materia orgánica (MO), \% & 89,49 & 88,35 \\
\hline Nitrógeno (N), \% & 1,32 & 2,13 \\
\hline Fósforo (P), \% & 1,12 & 1,79 \\
\hline Potasio (K), \% & 0,69 & 1,34 \\
\hline Calcio (Ca), \% & 0,39 & 0,61 \\
\hline Magnesio (Mg), \% & 0,33 & 0,52 \\
\hline Sodio (Na), \% & 0,02 & 0,04 \\
\hline Fierro (Fe), ppm & 1256,00 & 1224,00 \\
\hline Cobre (Cu), ppm & 34,00 & 47,00 \\
\hline Zinc (Zn), ppm & 49,00 & 74,00 \\
\hline Manganeso (Mn), ppm & 480,00 & 1326,00 \\
\hline Boro (B), ppm & 15,00 & 15,00 \\
\hline Plomo (Pb), ppm & 2,20 & 2,93 \\
\hline Cadmio (Cd), ppm & 0,53 & 0,68 \\
\hline Cromo (Cr), ppm & 21,18 & 10,85 \\
\hline
\end{tabular}

Cuadro 3. Características fisicoquímicas del biocarbón antes y después de ser activado con biol de estiércol de ganado vacuno.

\begin{tabular}{|c|c|c|c|c|}
\hline \multirow{3}{*}{ Parcela } & \multicolumn{4}{|c|}{ ALTURA (cm) } \\
\cline { 2 - 5 } & \multicolumn{4}{|c|}{ Días de plantación } \\
\cline { 2 - 5 } & $\mathbf{1}$ & $\mathbf{3 0}$ & $\mathbf{6 0}$ & $\mathbf{9 0}$ \\
\hline P1 & 6.60 & 7.74 & 8.68 & 16.60 \\
\hline P2 & 6.86 & 7.54 & 8.71 & 18.35 \\
\hline
\end{tabular}

Cuadro 4. Variación promedio de la altura de plantas de huayruro según los días de plantadas en P1 y P2.

\begin{tabular}{|c|c|c|c|}
\hline 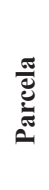 & 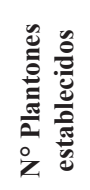 & 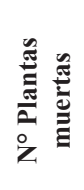 & \\
\hline P1 & 126 & 0 & 100 \\
\hline P2 & 126 & 9 & 93 \\
\hline
\end{tabular}

Cuadro 5. Porcentaje de supervivencia de plantas de O. schunkei, en P1 y P2, sin considerar el efecto borde a los 90 días de plantadas. tadas con otros elementos que se muestran en el Cuadro 1.

Los cuescos de palma aceitera que fueron utilizados como materias primas para fabricar el biocarbón, presentan concentraciones altas de Fe (493 ppm) y Mn (151 ppm) y valores bajos de N (1,13\%), P (0,47\%) y K (0,36\%), tal como se aprecia en el Cuadro 2.

\section{Caracterización fisicoquímica de biocarbón y biocarbón enriquecido}

Con respecto al biocarbón obtenido por medio de la pirólisis y enriquecido mediante su inmersión en biol de vacaza, los datos muestran incrementos en los macronutrientes como $\mathrm{N}$ de 1,32 a $2,13 \%$; de $\mathrm{P}$ de 1,12 a $1,79 \%$ y $\mathrm{K}$ de 0,69 a 1,34\%, y disminución leve en los valores de $\mathrm{pH}$ de 7,72 a 7,67; materia orgánica de $89,49 \%$ a $88,35 \%$ y Fe de 1256 ppm a 1224 ppm, correspondiendo al biocarbón y biocarbón enriquecido en vacaza respectiva- 
mente, los mismos que están reportados en el Cuadro 3.

\section{Comparación de la altura y porcentaje de su- pervivencia de $O$. schunkei}

En el caso del crecimiento en altura las plantas de O. schunkei, fueron analizadas con la prueba estadística de $t$ student. Con un $95 \%$ de confianza y $\alpha=0,05$. La prueba indicó que no existe diferencia significativa entre tratamientos a los 90 días (Cuadro 4).

\section{Crecimiento y supervivencia de las plantas de O. schunkei}

El porcentaje de supervivencia se evaluó considerando el efecto de borde. La plantación establecida en la parcela sin tratamiento presentó una supervivencia de $93 \%$. En las parcelas con tratamiento la supervivencia de plantones a los 90 días fue del $100 \%$ dado que la mortalidad se concentró en los bordes no evaluables (Cuadro 5).

\section{Discusión}

\section{Caracterización del biol de vacaza}

Si consideramos los valores determinados y mostrados en el Cuadro 1, de los elementos principales como: $\mathrm{pH}(3,5)$, Materia orgánica (33,16 g.L $\left.\mathrm{L}^{-1}\right)$, Nitrógeno-N total (994 mg.L $\left.\mathrm{L}^{-1}\right)$,
Fósforo-P total (336,31 mg.L-1 $)$, Potasio-K total (1 325 mg.L $\mathrm{L}^{-1}$ ), y estos resultados los comparamos con los obtenidos por Peralta et al. (2016), quienes también elaboraron un biol de ganado vacuno, el pH $(4,02)$ resultó ligeramente inferior, así como para N (4 200 mg.L $L^{-1}$ ) y K (17 200 mg. $\left.\mathrm{L}^{-1}\right)$. Estos resultados se pueden explicar en base al tipo de alimento recibido por los vacunos, pues los utilizados en este estudio, procedieron de animales alimentados en establo con alimento balanceado y pasto verde como complemento.

\section{Caracterización de los cuescos de palma aceitera}

Los resultados referentes a los análisis efectuados a los cuescos, mostrados en el Cuadro 2, nos indican elementos importantes para contribuir con nutrientes al suelo, sobre todo referido a los minerales. El valor para $\mathrm{N}$ $(1,13 \%)$ de los cuescos es mayor que el reportado por Muñoz (2013), quien reporto 0,9\% y $0,74 \%$, pero menor al $1,9 \%$ reportado por Díaz y Durán (2008), citados por Agudelo (2017). En cuanto al P (0,47\%), es mayor al 0,35\% reportado por Nizamuddin et al. (2016), y para K $(0,36 \%)$, es mayor al indicado por Navarrete et al. (2005), quienes reportaron $0,03 \%$. Estos resultados procedentes de los cuescos

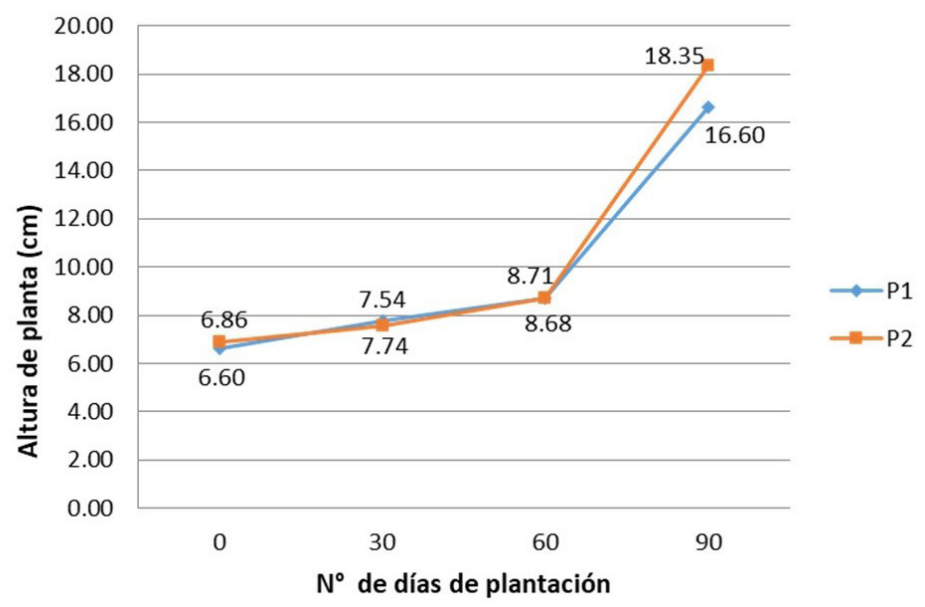

Figura 1. Altura total de los plantones de O. schunkei en P1 y P2 a los 0, 30, 60 y 90 días después de la plantación. 
obtenidos en Pucallpa frente a los reportados en otros estudios se pueden atribuir a factores edafo-climáticos, manejo de fertilización, tipo de suelo, y hasta la procedencia de las semillas del cual se originaron las plantas.

\section{Adaptación de las plantas de O. schunkei}

El Cuadro 4, nos muestra la característica de la altura lograda por las plantas de O. schunkei, mostrando una ligera ventaja en casi todas las mediciones que se hizo en la altura, destacando a los 90 días alturas de 16,60 y 18,35 cm en P1 y P2 respectivamente, atribuyendo a la aplicación del biocarbón activado en biol de estiércol de ganado vacuno dichos efectos. Esto es coherente con Situmeang et al. (2015), quienes investigaron la influencia de la aplicación del biocarbón de bambú en la altura de maíz y obtuvieron efectos similares. Asimismo, Espíritu (2009), en Satipo-Perú, investigó el efecto de la fertilización en la altura de plantas de Ormosia coccinea (Aubl.) Jacks., para lo cual aplicó tres diferentes dosis de NPK más un control, siendo estas de $100 \mathrm{~g}$ de NPK (10-30-10), con $150 \mathrm{~g}$ de NPK (15-45-15), y con $50 \mathrm{~g}$ de NPK (5-15$5)$, alcanzando a los siete meses con la primera dosis una altura media de $43,45 \mathrm{~cm}$; con la segunda dosis logró $34,10 \mathrm{~cm}$ y la tercera dosis de $32,80 \mathrm{~cm}$ de altura.

La Figura 1, describe la altura en $\mathrm{cm}$, de las plantas de O. schunkei, donde se observa que las plantas de la P2, tienen una leve ventaja sobre las de la P1, como consecuencia de la aplicación del biocarbón de cuescos de palma aceitera activado en biol de estiércol de ganado vacuno.

\section{Ganancia de altura de $O$. schunkei por efecto de los tratamientos}

El valor de $\mathrm{P}$ determinado para la altura de las plantas fue de 0,09 , el cual resultó mayor que el $\alpha=0,05$, demostrando que no existió diferencia significativa entre las medias de los tratamientos, es decir estadísticamente la ganancia de altura, no está influenciada por la aplicación del biocarbón. Sin embargo, la ganancia de altura por plantas con aplicación de biocarbón enriquecido, ha sido manifestada por Quispe (2019), quien utilizó biocarbón ac- tivado en caldo de humus en una proporción del 4\% (T2), en el cultivo de la hortaliza denominado canónigo (Valerianella locusta (L). Laterr.), con el cual logró una altura de planta de $11,99 \mathrm{~cm}$, atribuyendo a que el biochar mejora la disponibilidad de nutrientes, como también otras características del suelo que mejoran la fertilidad y por consecuente el rendimiento de los cultivos.

El efecto del biocarbón en combinación con un fertilizante sintético (Yaramila Complex), para promover el crecimiento de banano (Musa paradisiaca L.), fue reportado por Martínez et al. (2019), quienes indicaron que el biocarbón no solo mejora las propiedades del suelo, sino que también contribuye a mejorar el desempeño fisiológico de las plantas.

Por su parte Valarezo et al. (2016), quienes establecieron plantaciones de pachaco (Schizolobium parahyba (Vell.) S.F. Blake) y melina (Gmelina arborea Roxb. ex Sm.) durante 2 años, utilizando biocarbón en dosis de 0,3 y 6 t.h $\mathrm{h}^{-1}$, lograron en el pachaco con y sin fertilización una altura promedio de 6,49 y 3,91 m, respectivamente, con una diferencia de $2,58 \mathrm{~m}$, equivalente a $66 \%$. Por su parte, la melina con y sin fertilización alcanzó una altura promedio de 7,02 y 4,58 m, respectivamente, con una diferencia de $2,44 \mathrm{~m}$, lo que equivale a $53 \%$; por lo cual concluyeron que esta enmienda tuvo un efecto tardío en el crecimiento de las especies, atribuyendo a que la composición de los grupos superficiales es una característica importante para la exitosa utilización del biocarbón.

\section{Porcentaje de supervivencia}

En la Cuadro 5, se puede observar que hay mayor porcentaje de supervivencia de las plantas de O. schunkei, atribuido de modo directo a la aplicación del biocarbón. Las ventajas que se logra con la aplicación del biocarbón o biochar al suelo, ya han sido discutidas por Atkinson et al. (2010) y Glaser et al. (2001), citados por Situmeang et al. (2015), al manifestar que el biochar puede mejorar la fertilidad del suelo y ser capaz de recuperar la calidad de suelos degradados. Según Situmeang et al. (2015), el biochar puede mejorar la calidad del suelo de varias maneras, así puede lograr una mayor po- 
rosidad, un gran volumen y disponibilidad de agua, aumentar la capacidad de intercambio de $\mathrm{pH}$, C-orgánico, fósforo, potasio y capacidad de intercambio catiónico, reduciendo la lixiviación de nitrógeno y aumentando la actividad de las poblaciones microbianas.

Del mismo modo Espíritu (2009), experimentó el efecto de la fertilización en la plantación de Ormosia coccinea, aplicando 3 diferentes dosis de NPK más un control, siendo estas de $100 \mathrm{~g}$ de NPK (10-30-10), con $150 \mathrm{~g}$ de NPK (15-45-15), y, con 50 g de NPK (5-15-5), con las cuales logró una supervivencia a los 7 meses del 100\%.

Drake et al. (2015), experimentaron el uso de biocarbón en dosis de 0, 1, 3 y 6 t.h ${ }^{-1}$, aplicando al suelo y sembrando especies forestales de semilla directa en tres sitios en el oeste de Victoria, Australia, obteniendo como resultados que el Biochar aumentó el número de especies presentes y el conteo de tallos de $\mathrm{Eu}$ calyptus (Myrtaceae), mientras que disminuyó el conteo de tallos de Acacia (Fabaceae), concluyendo que el biocarbón tiene el potencial de beneficiar positivamente las actividades de reforestación, pero las respuestas específicas del sitio y del biocarbón de suelo-planta requieren una investigación específica.

En cuanto a la supervivencia, un dato importante es la relación altura/materia seca, la cual es indicada por Gomes et al. (2002), citados por Alonso et al. (2015), la cual resulta de gran utilidad para predecir el potencial de supervivencia de las plántulas en el campo, a pesar de no ser comúnmente usado como un índice para evaluar el patrón de calidad en fase de establecimiento, interpretándose que cuanto menor es este índice, más lignificada está la planta y mayor deberá ser la capacidad de supervivencia; por ello, para evaluar en el futuro, el porcentaje de supervivencia de la O. schunkei, será necesario tomar los datos de cantidad de materia seca producida por la parte aérea de las plantas.

\section{Conclusiones}

La aplicación de biocarbón de cuescos de palma aceitera enriquecido en biol de vacaza, tuvo un efecto positivo en el crecimiento y porcentaje de supervivencia de las plantas de $O$. schunkei, tal como lo demuestran los resultados obtenidos.

El alto porcentaje de supervivencia evitaría las operaciones de recalce, de mantenerse las condiciones iniciales de supervivencia, lo que tendría efectos muy positivos en los costos de mantenimiento de la plantación forestal durante el primer año.

El uso de biocarbón de cuescos de palma aceitera y enriquecido en biol de estiércol de ganado vacuno, es una alternativa viable para promover el crecimiento de O. schunkei.

Al comprobar en esta primera etapa de adaptación de O. schunkei, por el efecto promotor del biocarbón activado en biol de vacaza, se puede extender hacia otras especies forestales.

Finalmente, se recomienda continuar con los estudios de desarrollo y crecimiento de las plantas de O. schunkei para asegurar su total adaptación y crecimiento en el terreno experimental.

\section{Agradecimientos}

El presente trabajo se ha realizado gracias al financiamiento del Programa de Desarrollo Forestal Sostenible, Inclusivo y Competitivo en la amazonia peruana del SERFOR.

A los miembros de la Comunidad Nativa de San Francisco, ubicada en el distrito de Yarinacocha, provincia de Coronel Portillo, región de Ucayali, por su apoyo al habernos cedido el terreno para desarrollar la fase experimental del presente proyecto.

A los miembros del equipo investigador, por su constante apoyo y acompañamiento físico y moral, en esta ardua y difícil labor de generar conocimiento científico, pero que al mismo tiempo otorga gran satisfacción, al aportar un grano de arena para que el país salga adelante, pues la investigación nos permitirá enrumbarnos por el camino del desarrollo.

Al Ing. Leoncio Julio Ugarte Guerra, por la supervisión del experimento en campo y los consejos y apoyo en la evaluación estadística. 


\section{Bibliografía}

Agudelo Alvarez, BL. 2017. Estandarización de proceso de producción de carbón activado por activación física a partir de cuesco de palma a escala industrial (Tesis de pregrado). Bogotá C.D., Colombia, Fundación Universidad de América. 90 p.

Alonso López, M; Arteaga Crespo, Y; Geada López, G; García Quintana, Y; Carballo Abreu, L; Castillo Martínez, I. 2015. Características de sustratos orgánicos acondicionados con biocarbón.: Influencia en la calidad de plantas de Talipariti elatum (Sw.) Fryxell cultivada en tubetes. Revista Cubana de Ciencias Forestales: CFORES 3(1):1.

Cochrane, TP. 1982. Caracterización Agroecológica para el desarrollo de pasturas en suelos ácidos de América tropical. Cali, Colombia CIAT. 44p.

Drake, JA; Carrucan, A; Jackson, WR; Cavagnaro, TR; Patti, AF. 2015. La aplicación de biocarbón durante la reforestación altera las especies presentes y la química del suelo. Science of the Total Environment 514:359-365.

Emery Genes, EJ. 2014. Destilación secundaria de alquitranes generados en la gasificación de cuesco de palma africana (Tesis de posgrado). Medellín, Colombia, Universidad Nacional de Colombia. 56 p.

Escola, JM; Aguado, J; Serrano, DP; Briones, L. 2014. Transportation fuel production by combination of LDPE thermal cracking and catalytic hydroreforming. Waste management 34(11):2176-2184.

Espíritu Caballero, AD. 2009. Plantación de Ormosia coccinea Jack en fajas y fertilización con tres dosis NPK. Unión Capiri - Satipo (Tesis de pregrado). Satipo, Perú, Universidad Nacional del Centro del Perú. 34 p.

Guerrero, F; Serrano-Martínez, E; Tantaleán, M; Quispe, M; Casas, G. 2012. Identificación de parásitos gastrointestinales en primates no humanos del zoológico parque natural de $\mathrm{Pu}$ callpa, Perú. Revista de investigaciones veterinarias del Perú 23(4):469-478.
Gutiérrez, AH; Peña, LB; Díaz, JGH. 2018. Obtención de biogás mediante la fermentación anaerobia de estiércol. Revista Estudiantil AGRO-VET 2(2):185-191.

Martínez, ST; Guerrero, JNQ; Batista, RMG. 2019. Determinación de la dosis óptima de biocarbón como enmienda edáfica en el cultivo de banano (Musa $\times$ paradisiaca L.) Clon Williams. Revista Científica Agroecosistemas 7(3), 134-141.

Muñoz Millán, J. 2013. Gasificación térmica de cuesco de palma: Pirolisis, Reformado de vapor y Gasificación con oxígeno. Tesis de posgrado. Bogotá DC, Colombia, Universidad de los Andes. 57 p.

Navarrete, LF; Giraldo, L; Baquero, MC; Moreno, JC. 2005. Carbón activado: Efecto del lavado con ácido sulfúrico del precursor lignocelulósico, cuesco de palma africana, sobre los procesos de carbonización y activación. Revista Colombiana de Química 34(1):67-38.

Nizamuddin, S; Shrestha, S; Athar, S; Si, BA; Ahmar SM. 2016. A critical analysis on palm kernel shell from oil palm industry as a feedstock for solid char production. Reviews in Chemical Engineering 32(5):489-505.

Peralta Veran, L; Juscamaita Morales, J; Meza Contreras, V. 2016. Obtención y caracterización de abono orgánico líquido a través del tratamiento de excretas del ganado vacuno de un establo lechero usando un consorcio microbiano ácido láctico. Ecología Aplicada 15(1):110.

Quispe Patzi, JM. 2019. Evaluación de tres niveles de biochar en el cultivo de canónigo (Valerianella locusta) bajo ambiente atemperado en la Estación Experimental de Patacamaya. Tesis de pregrado. La Paz, Bolivia, Universidad Mayor de San Andrés.

Ruíz, P, O; Davey, Ch, B. 2005. Micorrizas arbusculares en ultisoles de la Amazonía peruana. Folia Amazónica 14(2):57-74.

Situmeang, YP; Adnyana, IM; Subadiyasa, INN y Merit, IN. 2015. Efecto de la dosis Biochar Bamboo, Compost y Phonska sobre el cre- 
cimiento del maíz (Zea mays L.) en tierras secas. Revista Internacional de Ciencia Avanzada, Ingeniería y Tecnología de la Información 5(6):433-439.

Valarezo, C; Villamagua, MA; Mora, RM; Maza, H; Wilcke, W; Nieto, C. 2016. Respuesta del pachaco (Schizolobium parahybum Vell. Conc) y la melina (Gmelina arborea Roxb.) a la aplicación de biocarbón y fertilización en el sur de la Amazonia Ecuatoriana. Bosques Latitud Cero 6(1):1-32. 